\title{
Angiosarcoma primario uterino: revisión de la literatura y presentación de caso
}

\author{
Diana Sandoval M. ${ }^{1}$, Carlos García R. ${ }^{1}$, Magda López A. a, Ernesto García A. ${ }^{1}$ \\ 1 Departamento de Patología, Facultad de Salud, Universidad Industrial de Santander (Bucaramanga-Colombia). \\ a Enfermera Profesional. Grupo de Patología Estructural y Funcional (PATUIS). Universidad Industrial de Santander, \\ Bucaramanga-Colombia
}

\section{RESUMEN}

Antecedentes: Los angiosarcomas son infrecuentes y corresponden a menos del $1 \%$ de tumores de tejidos blandos, pueden presentarse en cualquier localización fundamentalmente en la piel. El angiosarcoma primario uterino fue por primera vez reportado en 1902 por Silberberg con informes ulteriores esporádicos que suman un total de 33 casos incluyendo el presente. Objetivo: Exponer un caso diagnosticado en el Departamento de Patología de la Universidad Industrial de Santander (Bucaramanga-Colombia), y revisar la literatura de los casos previamente descritos. Caso clínico: Paciente de 25 años, con 2 años de evolución de menometrorragias y dolor pélvico crónico. Se realiza ablación endometrial encontrándose tumor maligno de endometrio pobremente diferenciado, infiltrante, el cual no es posible categorizar. Se efectúa una histerectomía ampliada, salpingooforectomía bilateral, linfadenectomía pélvica y omentectomía. El examen microscópico reveló lesión tumoral pobremente diferenciada compatible con un angiosarcoma uterino de alto grado.

\section{PALABRAS CLAVE: Angiosarcoma uterino primario, inmunohistoquímica}

\section{SUMMARY}

Background: Angiosarcomas are rare and account for less than 1\% of soft tissue tumors can occur at any location primarily in the skin. The primary uterine angiosarcoma was first reported in 1902 by Silberberg with sporadic subsequent reports, with a total of 33 cases including the present. Objective: To present a case diagnosed in the Department of Pathology, Universidad Industrial de Santander (Bucaramanga, Colombia) and review the literature of the cases previously described. Case report: A 25 years old woman, with 2 years of chronic pelvic pain and menometrorrhagia. Endometrial ablation is performed and an endometrial malignancy was found, poorly differentiated and infiltrating, which is not possible to categorize. It makes an extended hysterectomy, bilateral salpingo-oophorectomy, pelvic lymphadenectomy, and omentectomy. Microscopic examination revealed poorly differentiated tumor, compatible with a high-grade uterine angiosarcoma.

\section{KEY WORDS: Primary uterine angiosarcoma, immunohistochemestry}

\section{INTRODUCCIÓN}

En nuestro medio los tumores malignos del cuerpo uterino representan el $3,1 \%$ de las neoplasias que afectan las mujeres, siendo el $91,9 \%$ de estas derivadas del endometrio y el $8,1 \%$ restantes lesiones miometriales, básicamente sarcomas (1). El angiosarcoma primario uterino fue por primera vez descrito en la literatura en 1902 por Silberberg (2), con reportes ulteriores esporádicos que suman 
un total de 33 casos incluyendo el presente trabajo.

El angiosarcoma es definido como una neoplasia mesenquimal originada de las células endoteliales presentes en los vasos sanguíneos y que pueden tener en su localización en el cuerpo uterino, una apariencia macroscópica polipoide o ser completamete intramurales con zonas de hemorragia, necrosis e incluso formaciones quísticas (3). Están compuestos por canales vasculares anastomosantes los cuales se encuentran tapizados por células tumorales cuboides que exhiben moderada a marcada atipia y que presentan altos conteos mitóticos (2-6), dichas zonas pueden alternar con áreas sólidas que muestran células en un espectro que van desde ahusadas hasta epitelioides, que dependiendo de la representación de dichas áreas en la totalidad del tumor pueden representar la subvariante de angiosarcoma epitelioide $(4,7)$. Son considerados tumores altamente agresivos, cuyo diagnóstico definitivo requiere demostración por inmunohistoquímica y/o microscopia electrónica $(2-5,8,9)$.

El objetivo de esta comunicación es exponer un caso diagnosticado histopatológicamente en el Departamento de Patología de la Universidad Industrial de Santander, en una muestra de patología enviada por el Hospital Universitario de Santander en el 2010, y hacer una revisión de la literatura de esta entidad, dada la baja frecuencia de su diagnóstico y a la escasa información al respecto, siendo este el primer caso reportado en Colombia y Latinoamérica y el segundo reportado en una paciente de raza hispana.

\section{Caso clínico}

Paciente mujer de 25 años, gesta 1, para 0, con cuadro de 2 años de evolución caracterizado por menometrorragias y dolor pélvico crónico, manejado con hormonoterapia oral, colocación de dispositivo intrauterino liberador de levonorgestrel y biopsia endometrial en 3 ocasiones, con reportes negativos para malignidad. Antecedentes patológicos de déficit de factor XIII en manejo con crioprecipitados trimestrales. Por persistencia del cuadro clínico, se ordena ecografía transvaginal que evidencia un endometrio de $43 \mathrm{~mm}$, monolaminar, ovarios de tipo polimicroquístico. Es programada para ablación endometrial en septiembre de 2010, encontrándose poliposis endometrial múltiple y presentando como complicación perforación uterina con manejo expectante. Los hallazgos histopatológicos revelaron tumor maligno pobremente diferenciado e infiltrante, el cual no es posible categorizar y se recomienda realización de inmunohistoquímica. Es evaluada por el Servicio de gineco-oncología quienes realizan histerectomía ampliada, salpingooforectomía bilateral, linfadenectomía iliaca y omentectomía, sin complicaciones posquirúrgicas. El estudio histopatológico reveló lesión tumoral dependiente del cuerpo uterino que infiltra la serosa extendiéndose al parametrio derecho (Figura 1). El estudio con microscopia de luz evidenció lesión tumoral pobremente diferenciada, conformada por células con alto pleomorfismo de núcleos rendondeados con cromatina dispersa y nucleolo prominente con conteo mitótico de 3 por 10 campos de alto poder (HPF), que se disponen de manera discohesiva con formación de estructuras vasculares anastomosantes y focos de hemorragia (Figura 2) se realizaron estudios de inmunohistoquímica con positividad de las células tumorales para CD31, CD34, Factor VIII (Figura 3) y negatividad para AE1AE3, CK20, CK7, EMA y receptores de estrógeno (Figura 4), con diagnóstico definitivo de angiosarcoma uterino de alto grado. La paciente 24 meses después del diagnóstico recibió radio y quimioterapia y no ha presentado recaídas.

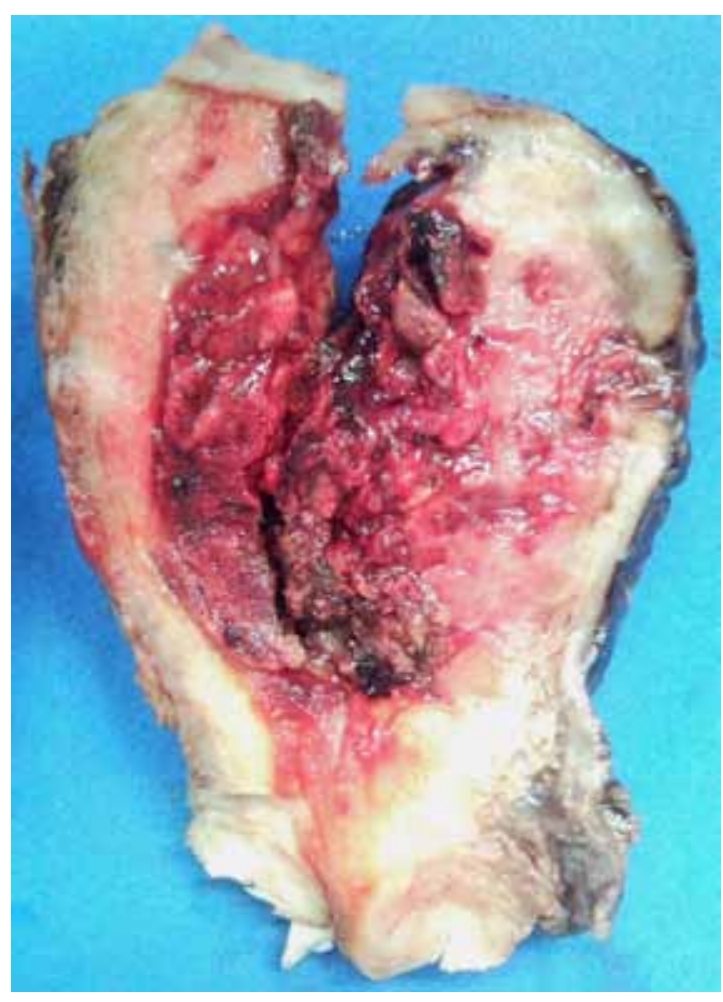

Figura 1. Aspecto macroscópico de la cavidad endomiometrial en su pared posterior lesión tumoral que depende del cuerpo uterino de $5 \times 4 \mathrm{~cm}$, de apariencia polipoide que infiltra la pared miometrial y compromete hasta la serosa, extendiéndose al parametrio derecho. 


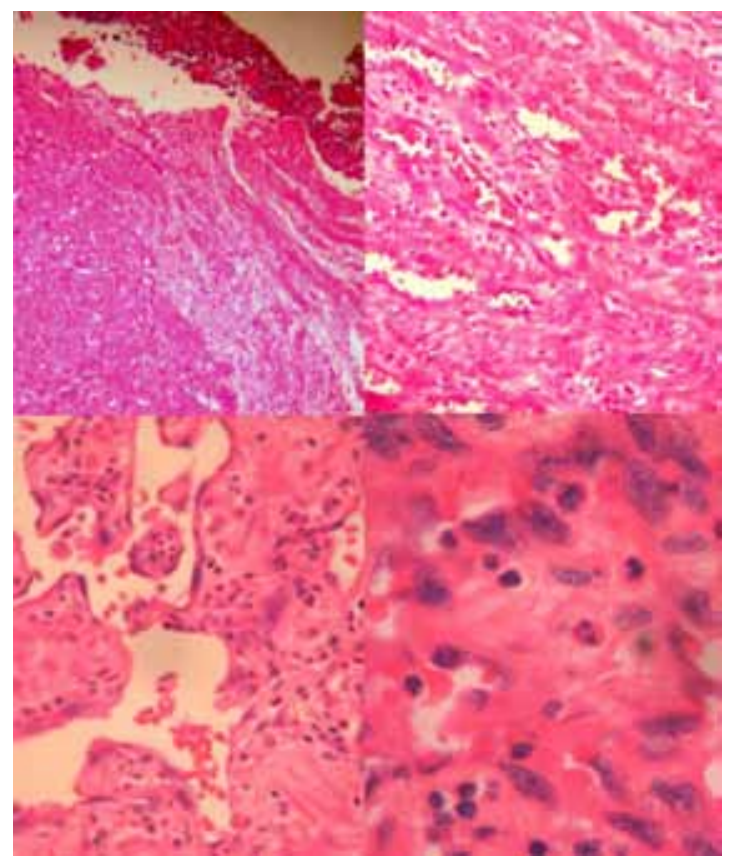

Figura 2. Aspecto histológico reveló lesión tumoral pobremente diferenciada conformada por células con alto pleomorfismo de núcleos rendondeados con cromatina dispersa y nucleolo prominente con conteo mitótico de 3 por 10 HPF, que se disponen de forma discohesiva con formación de estructuras vasculares anastomosantes y focos de hemorragia.

\section{DISCUSIÓN}

Los angiosarcomas son lesiones infrecuentes que corresponden a menos del $1 \%$ de los tumores de tejidos blandos y que pueden presentarse en cualquier localización, fundamentalmente en la piel y tejido celular subcutáneo de la cara y del escalpo de adultos mayores $(2,8,9,10)$. La localización somática es inusual y se han reportado casos esporádicos de localización visceral en pulmón, glándula mamaria, pleura e hígado (2). En el cuerpo uterino se han publicado 33 casos (2,6-9,11-14), incluido el presente, con características epidemiológicas específicas que incluyen presentación en mujeres en su mayoría posmenopáusicas con un rango de edad que oscila entre 17 y 81 años (edad promedio de 52 años); 7 casos, incluido el presente, han sido descritos en mujeres premenopausicas y con mayor sobrevida.
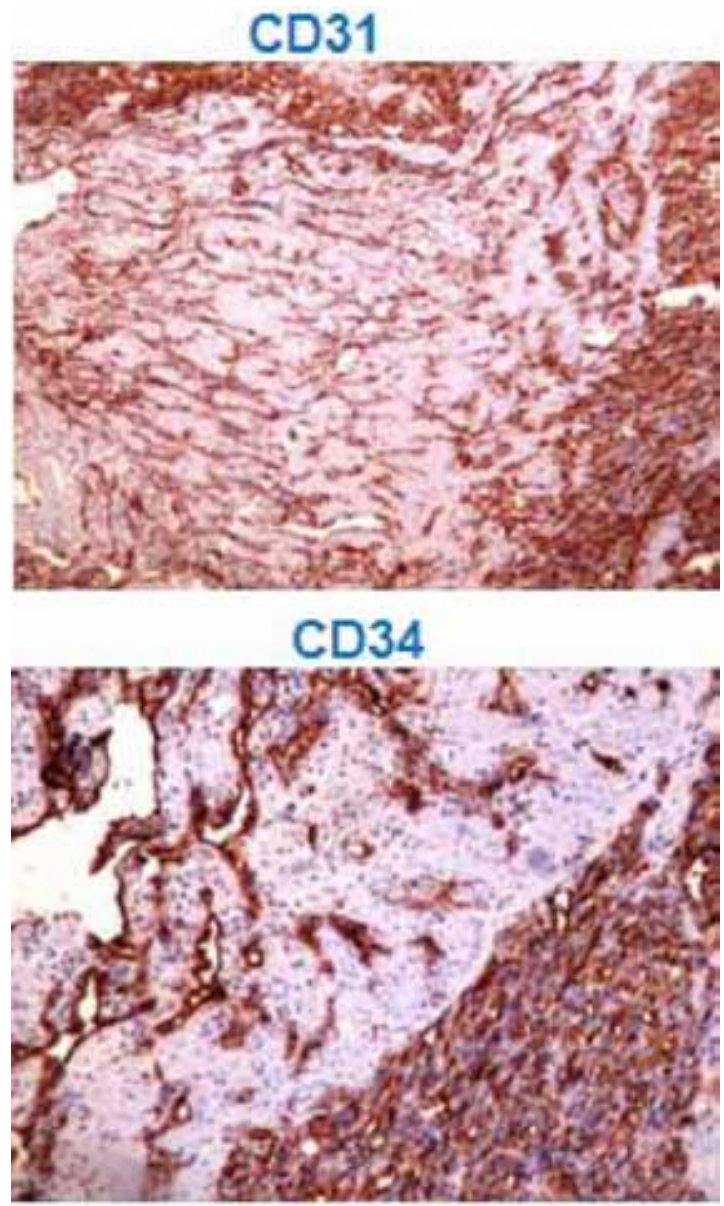

FACTOR VIII

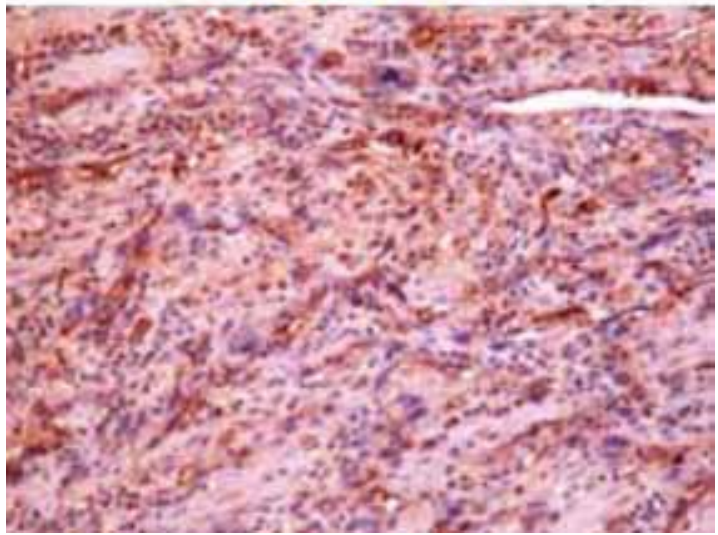

Figura 3. Positividad de las células tumorales para el CD31, CD34 y factor VIII. 
AE1/AE3, CK7, CK20

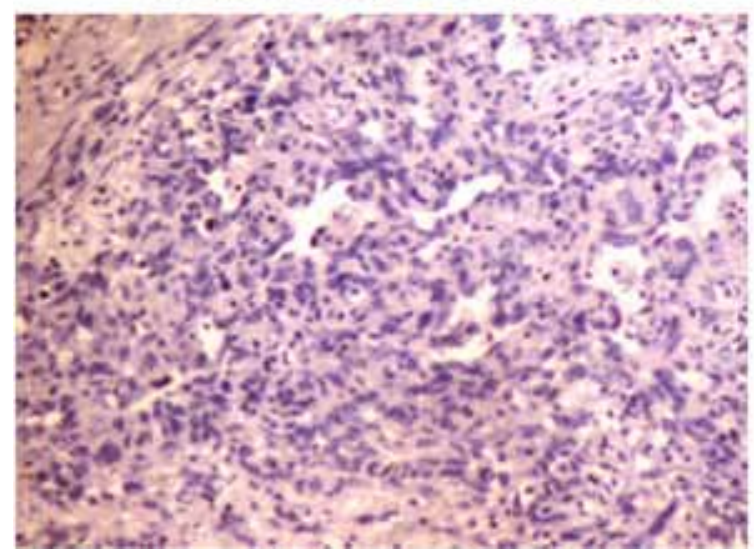

Rc ESTRÓGENOS

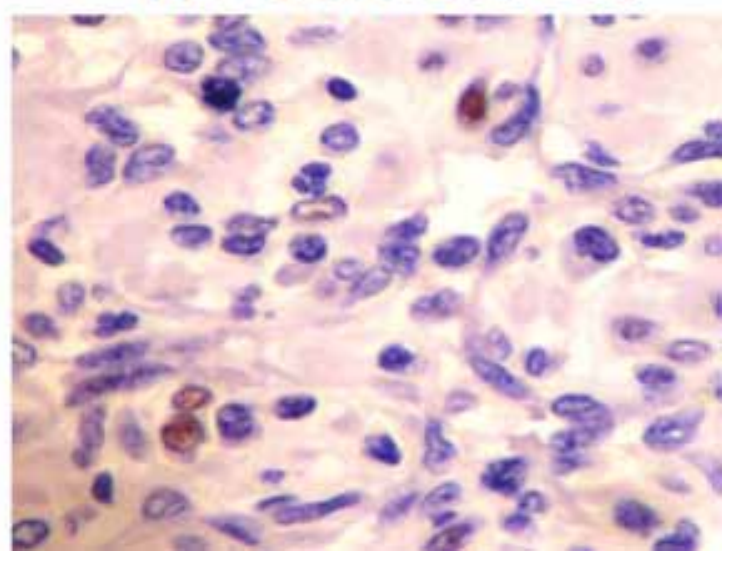

Figura 4. Las células tumorales son negativas para citoqueratinas y receptores de estrógenos

Macroscópicamente han sido descritos como masas de 4 a $30 \mathrm{~cm}$ con infiltración miometrial profunda, que presentan microscopicamente, las mismas características de las neoplasias malignas de derivación endotelial. Se distribuyen en un espectro que va desde el clásico patrón con formación de canales vasculares, tapizadas por células con pleomorfismo de leve a moderado e hipercromasia, hasta su contraparte indiferenciada de células ahusadas que sugiere fibrosarcomas o células epitelioides con nucléolo prominente que puede sugerir carcinoma o incluso melanomas; por eso es necesario un adecuado panel de inmunohistoquímica para definir el origen de estas lesiones mesenquimales cuyas características con las microscopia óptica y hematoxilina-eosina pueden superponerse (15), y se deben descartar lesiones más frecuentes en esa localización como leiomiosarcoma, tumor mulleriano mixto o sarcoma endometrial que pueden tener marcada vascularización $(2,8)$, confirmándose su origen con anticuerpos específicos de células endoteliales tales como CD31, CD34 y factor VIII y descartando la actividad de otros marcadores, como citoqueratinas (AE1/AE3, CK7, CK20), marcadores de músculo, (actina, desmina, H-caldesmon) EMA, S-100, receptores de estrogénos en las células tumorales $(2,4,7,16)$. A nivel de la ultraestructura puede confirmarse la presencia de cuerpos de Weibel-Palade en el citoplasma de las células tumorales $(2,8)$.

Este es el segundo caso reportado en menores de 30 años, el primero fue descrito en el año 1979 por Ehrmann y Griffiths (6), en una paciente de 17 años, quien al momento del diagnóstico presentaba extensión a través de la pared uterina al ovario iz- quierdo; posteriormente y a pesar de los tratamientos quirúrgicos y adyuvantes con radioterapia fallece. También es el segundo reporte hecho en una mujer de origen latinoamericano y el primer diagnóstico de esta lesión hecho en Sudamérica, la paciente 18 meses después del diagnóstico recibió radioterapia y no ha presentado recaídas.

\section{CONCLUSIÓN}

A pesar de ser una patología infrecuente y de la temprana edad de la paciente, la presencia de atipias marcadas y la conformación de canales vasculares, sugieren que la lesión es un angiosarcoma al estudio de microscopía de luz. Son necesarios estudios de confirmación inmunohistioqumica o de microscopia electrónica que permitan confirmar la estirpe tumoral y descartar otras lesiones mas frecuentes en esta localización que puedan presentar un incremento de la proliferación vascular.

\section{REFERENCIAS}

1. Uribe JC, Meza EE. Incidencia de cáncer en el Área Metropolitana de Bucaramanga, 2000-2004. MedUNAB 2007;10:147-72.

2. Mendez LE, Joy S, Angioli R, Estape R, Penalver M. Primary uterine angiosarcoma. Gynecol Oncol 1999;75:272-6.

3. Fadare $\mathrm{O}$. Heterologus and rare homologous sarcome of the uterine corpus: a clinicopathologic review. Adv Anat Pathol 2011;18;60-74.

4. Kurman RJ, Ellenson LH, Ronnett BM. Blaustein's Pathology of the Female Genital Tract. 5ta ed. Springer. 2011, p.453-527. 
5. Moinfar F, Azodi M, Tavassoli FA. Uterine sarcomas. Pathology 2007;39:55-71.

6. Ehrmann RL, Griffiths CT. Malignant hemangioendothelioma of the uterus. Gynecol Oncol 1979;8:376-83.

7. Olawaiye AB, Morgan JA, Goodman AK, et al. Epitheliod angiosarcoma of the uterus: a review of management. Arch Gynecol Obstet 2008;278:401-4.

8. Cardinale L, Mira M, Galli C, et al. Angiosarcoma of the uterus: report of 2 new cases with deviant clinicopathologic features and review of the literature. Ann Diagn Pathol 2008;12:217-21

9. Pina $\mathrm{C}$, Teixera $\mathrm{M}$, Torres $\mathrm{S}$, et al. Primary uterine angiosarcoma. Arquivos de Medicina 2008;22(4/5):123-5.

10. Fisher C, Montgomery EA, Thway K. Biopsy interpretation of soft tissue tumors. 1ra ed. Lippincott Williams \& Wilkins. 2011, p.381-446.

11. Pedowitz P, Felmus LB, Grayzel DM. Vascular tumors of the uterus. II. Malignant vascular tumors. Am J Obs- tet Gynecol 1955;69:1309-22.

12. Konishi $Y$, Sato H, Fujimoto T. A case of primary uterine: magnetic resonance imaging and computed tomography findings. Int J Gynecol Cancer 2007;17:280-4.

13. Schammel DP, Tavassoli FA. Uterine angiosarcomas: a morphologic and immunohistochemical study of four cases. Am J Surg Pathol 1998;22:246-50.

14. Morrel B, Mulder AF, Chadha S, Tjokrowardojo AJ, Wijnen JA. Angiosarcoma of the uterus following radiotherapy for squamous cell carcinoma of the cervix. Eur $J$ Obstet Gynecol Reprod Biol 1993;49:193-7.

15. Abeler VM, Nenodovic M. Diagnostic immunohistochemestry in uterine sarcomas: a study of 397 cases. Int $J$ Gynecol Pathol 2011;30:236-43.

16. Chowdhary ND, Malik TM, Laharwal MA, Kadri SM. Primary Angiosarcoma of ovary--A case report with review of literature. JK Science 2001;3:194-7 